\title{
Four-terminal organic solar cell modules with increased annual energy yield
}

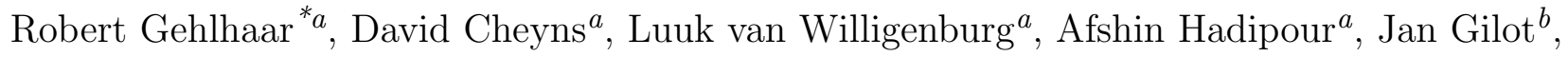 \\ Roshanak Radbeh ${ }^{c}$, and Tom Aernouts ${ }^{a}$ \\ ${ }^{a}$ IMEC, Kapeldreef 75, 3001 Leuven, Belgium; \\ ${ }^{b}$ Holst Centre, High Tech Campus 31, 5656 AE Eindhoven, Netherlands; \\ ${ }^{c}$ Solvay, MDA, Rue de Ransbeek 310, 1120 Brussels, Belgium
}

\begin{abstract}
The authors present experimental results on mechanically stacked organic solar modules and their advantage over standard tandem architectures. A four-terminal configuration of two single junction modules with complementary absorbing active layers uses the more efficient energy conversion of a tandem structure without the necessity of matching currents or voltages of electrically connected subcells. The presented combination of semitransparent and opaque solar cells consists of solution processed polymer-fullerene blends as active materials. A cost-effective mechanical scribing process is applied for the patterning of the deposited layers. The best devices have an efficiency of over $6.5 \%$ on an aperture area of $16 \mathrm{~cm}^{2}$ which equals a gain of $30 \%$ over the best single junction module fabricated by the same process. Optical simulations demonstrate a $32 \%$ increased annual energy output of a mechanically stacked device in comparison to a monolithic tandem structure using an equivalent geometry.
\end{abstract}

Keywords: Organic solar cells, bulk heterojunction, semitransparent solar cells, polymer solar cells, OPV, submodules, tandem

\section{INTRODUCTION}

Organic solar cell efficiencies are currently passing $12 \%$ achieved with devices of lab scale sizes. ${ }^{1}$ Although these efficiencies are still clearly exceeded by established silicon and other inorganic semiconductor technologies, ${ }^{2}$ the potentially low cost fabrication process brings organic photovoltaics into a competitive market position. Besides the low energy and material consumption in the production process, the unique properties of mechanical flexibility, light weight and semitransparent devices will lead to new applications of solar cells not accessible by common device architectures. A necessary step from lab records to customer products is the upscaling of the material synthesis, the device structures and fabrication process.

The most efficient organic solar cells are tandem structures which combine two complementary absorbing subcells in a serial connection and a monolithic architecture. ${ }^{3,4}$ In such configurations, the incident light is more efficiently harvested as photons with higher energy are absorbed by materials that generate a higher open circuit voltage. Since two or more devices are connected in series, the currents generated in the subcells have to be balanced. ${ }^{5}$ This requires a well-aligned light distribution in the tandem structure which makes the device potentially sensitive to changes in the spectrum and the angle of the incident light. We present a mechanically stacked device consisting of two semitransparent submodules that operate independently by using the same aperture. In such a four-terminal configuration, the dependencies on the incident light conditions are reduced compared to monolithic tandem devices as no current or voltage matching is required for an efficient operation. The two submodules are mechanically connected by a gluing process as it is identically used for an encapsulation by edge sealing. In this composition, the substrates and the hardened adhesive encapsulate the organic solar cells from oxygen and humidity. Although the samples presented within this work are entirely fabricated on rigid glass substrates, the fabrication processes can be also adapted to flexible polymer foils or other carrier compositions.

*Further author information: (Send correspondence to R.G.)

E-mail: gehlhaar@imec.be, Telephone: +32 (0) 16287715

Organic Photovoltaics XIV, edited by Zakya H. Kafafi, Paul A. Lane, Proc. of SPIE Vol. 8830,88300 I

(C) 2013 SPIE $\cdot$ CCC code: $0277-786 X / 13 / \$ 18 \cdot$ doi: $10.1117 / 12.2024023$ 


\title{
2. EXPERIMENTAL
}

\subsection{Materials and layer fabrication}

The organic solar cells presented within this paper are fabricated in an inverted architecture enabling the electron extraction at the bottom contact. Glass substrates of $0.7 \mathrm{~mm}$ thickness coated with an indium-tin-oxide (ITO) layer of $15 \Omega / \square$ sheet resistance are patterned by photolithography and wet-etching. These samples are cleaned in a sequence of detergent, de-ionized water, acetone and isopropanol, each step for 10min in an ultra sonic bath. An electron extraction layer of titanium oxide $\left(\mathrm{TiO}_{x}\right)$ is formed by a sol-gel process after spin coating a solution prepared following the recipe described in Ref. 6. A spin speed of $1000 \mathrm{rpm}$ results in a final film of $\mathrm{TiO}_{x}$ with a thickness of $3 \mathrm{~nm}$. The organic photoactive layers are prepared by spin coating at speeds from $600 \mathrm{rpm}$ to $1000 \mathrm{rpm}$ resulting in effective thicknesses of $70 \mathrm{~nm}$ to $100 \mathrm{~nm}$. For the top contacts two similar layer compositions are applied depending on the final application. For opaque monolitic devices, the electrodes are made of $10 \mathrm{~nm}$ molybdenum oxide $\left(\mathrm{MoO}_{3}\right)$ and $150 \mathrm{~nm}$ silver resulting in high reflectivity and low sheet resistance. In semitransparent devices the structure of $10 \mathrm{~nm} \mathrm{MoO} 3 / 10 \mathrm{~nm} \mathrm{Ag} / 50 \mathrm{~nm} \mathrm{MoO} 3$ is applied as top electrode ensuring a good balance of optical transmission and electrical charge extraction and charge transport with sheet resistances of $\mathrm{R}_{s}<10 \Omega / \square . .^{7}$ Both top contact configurations are prepared by thermal evaporation in a high vacuum chamber at pressures of $\mathrm{p}<5 \times 10^{-7}$ Torr on cooled substrates with temperatures of $\mathrm{T}<0^{\circ} \mathrm{C}$.

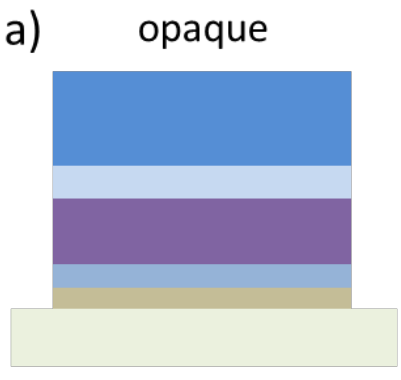

\section{b) semitransparent}
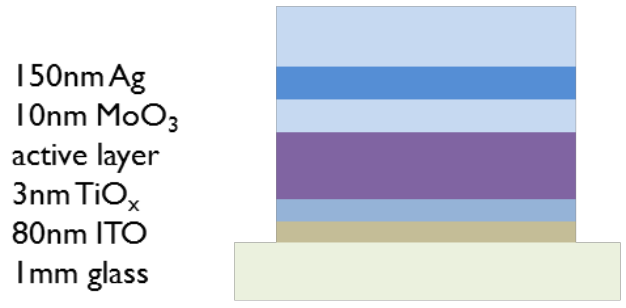

\author{
$50 \mathrm{~nm} \mathrm{MoO}_{3}$ \\ $10 \mathrm{nmAg}$ \\ $\mathrm{IOnm} \mathrm{MoO}_{3}$ \\ active layer \\ $3 \mathrm{~nm} \mathrm{TiO}$ \\ $80 \mathrm{~nm}$ ITO \\ I $\mathrm{mm}$ glass
}

Figure 1.

Structure and layer thicknesses of opaque (a) and semitransparent (b) OPV devices. The active material thickness varies between $70 \mathrm{~nm}$ and $100 \mathrm{~nm}$ depending on the selected composition. In all experiments the light is incident from the side of the glass substrate.

The photovoltaic characteristics were measured under nitrogen atmosphere using a Keithley 2602A source meter under $100 \mathrm{~mW} / \mathrm{cm}^{2}$ AM1.5 simulated illumination using an Abet Technologies Sun 2000 solar simulator with a $550 \mathrm{~W}$ Xenon arc lamp. The active areas of the devices are individually determined under an optical microscope.

As photoactive donor materials the two commercially available polymers PV10 and XPbing are used. PV10 is provided by Polyera Corp. and XPbing is supplied by Plextronics, Inc. Both polymers are delivered in combination with an acceptor in a ready-to-use ink to form a bulk-heterojunction after the spin coating process step. For optimal results no further film treatment is applied. The two material systems have been selected according to convenient fabrication applicability and due to their electrical and optical properties in OPV devices. The optical properties are depicted by the external quantum efficiency (EQE) in Fig.2 as measured in small area devices $\left(\mathrm{A} \approx 13 \mathrm{~mm}^{2}\right)$ with opaque thick Ag top contacts. PV10 shows a high EQE of over $50 \%$ over a spectral range from $350 \mathrm{~nm}$ to $700 \mathrm{~nm}$, while XPbing is generating extractable charges for absorbed light from $350 \mathrm{~nm}$ up to $900 \mathrm{~nm}$ wavelength with a slightly lower maximum efficiency compared to PV10.

In Table 1 devices in opaque and semitransparent geometry are compared for the materials systems of PV10 and XPbing. Despite the broader absorption range, the XPbing devices exhibit lower efficiencies than their PV10 counterparts. This result is based on the higher fill factors and higher open circuit voltages of the PV10 devices. At the time of investigation XPbing offered the best balance of ease of handling and electro-optical properties for a near-infrared solution-processable absorber. In the combination PV10-XPbing, the high performing PV10 bulkheterojunction system is used as the absorber for shorter wavelength range converting light of the whole visual 


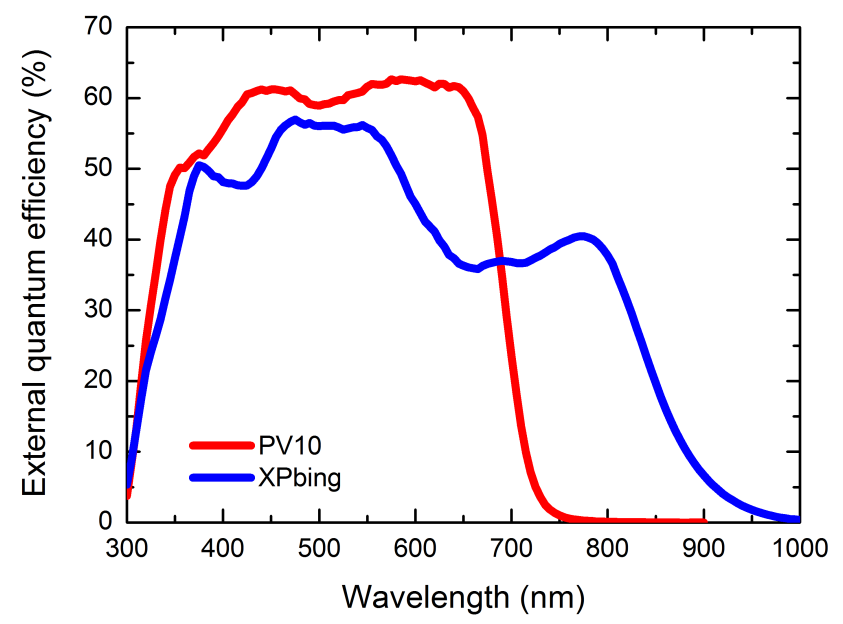

Figure 2.

External quantum efficiency of single opaque cells with $150 \mathrm{~nm}$ top contacts.

spectrum efficiently into extractable charges. In the most efficient devices, only the near-infrared absorption capabilities of XPbing from $700 \mathrm{~nm}$ to $950 \mathrm{~nm}$ are used, therefore only contribution to a small extend to the total power output of a mechanically stacked tandem configuration.

Table 1. Electrical properties of small area $\left(\mathrm{A} \approx 13 \mathrm{~mm}^{2}\right)$ monolithic PV10 and XPbing devices. Opaque devices with intransparent $150 \mathrm{~nm} \mathrm{Ag}$ top contacts are compared to semitransparent cells with $10 \mathrm{~nm} \mathrm{Ag} \mathrm{electrodes.} \mathrm{All} \mathrm{devices} \mathrm{have}$ been illuminated through the glass substrate.

\begin{tabular}{|c|c|c|c|c|c|}
\hline active material & configuration & Jsc $\left[\mathrm{mA} / \mathrm{cm}^{2}\right]$ & Voc $[\mathrm{mV}]$ & FF $[\%]$ & Eff $[\%]$ \\
\hline PV10 & opaque & 15.4 & 790 & 70 & 8.5 \\
\hline PV10 & semitransparent & 12.0 & 770 & 66 & 6.1 \\
\hline XPbing & opaque & 14.0 & 720 & 53 & 5.3 \\
\hline XPbing & semitransparent & 8.8 & 710 & 56 & 3.5 \\
\hline
\end{tabular}

\subsection{Module design and fabrication}

Large area devices within this work have been prepared on $5.5 \times 5.5 \mathrm{~cm}^{2}$ glass substrates with an aperture area of $4 \times 4 \mathrm{~cm}^{2}$ covered by ITO. This aperture area consists of 8 ITO stripes of about $5 \mathrm{~mm}$ width separated by a $70 \mu \mathrm{m}$ edged line. This design is the result of an optimization balancing the fabrication feasibility and the electrical losses within the transparent contacts. The module fabrication follows the description given in Sec. 2.1 with the addition of two patterning steps. The rather high sheet resistances of the applied transparent contacts prevent an efficient operation of single large area device of the size as used within this work. By a fragmentation of the total area in a stripe design and a serial connection of the individual subcells as shown in Fig. 3 the ohmic losses are limited to about $5 \%$ in comparison to more than $50 \%$ in an unpatterned design. The abbreviations in common for the patterning steps are $\mathrm{P} 1, \mathrm{P} 2$, and $\mathrm{P} 3$, where $\mathrm{P} 1$ is the photo lithographic process of the ITO lift-off, P2 is the removal of the organic film to enable the connection of neighboring subcells via their bottom and top contact, and P3 is the patterning of the top contact. While the P2 patterning step is applied directly after the organic layer deposition, the P3 marks the end of the film fabrication of a single module.

The patterning steps P2 and P3 are performed under inert atmospheric conditions in a nitrogen glovebox by 


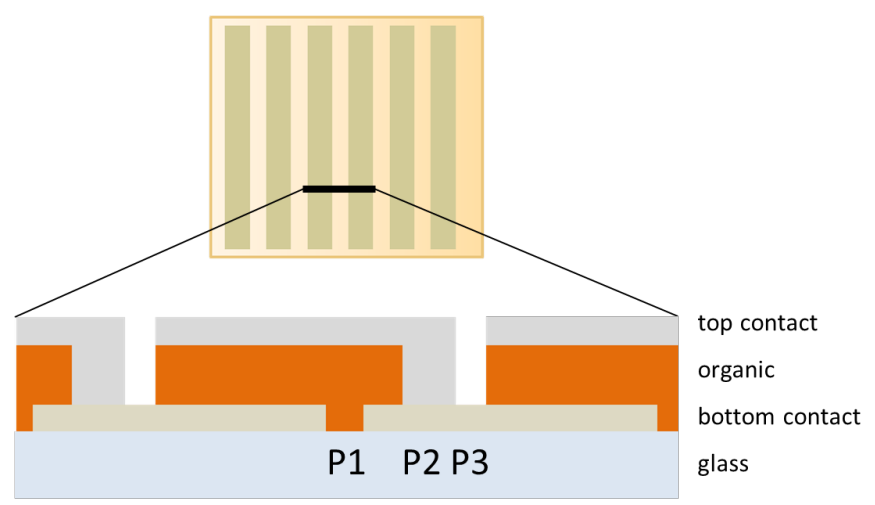

Figure 3.

Illustration of the patterning of the OPV modules in order to serially connect the single cells on a substrate. The cross section of two subcells depicts the three patterning steps P1, P2, and P3 that separate the bottom and top contacts of the neighboring cells. Via the P2 step cells are connected in series by contacting the Ag film and the ITO layer of the neighboring cell.

means of a mechanical scribing setup. This setup consists of a computer controlled xyz-stage with an attached metal blade. The setup allows for a positioning precision of about $10 \mu \mathrm{m}$. Figure 4 shows two optical microscope images of the patterned area of an OPV module. In Fig. $4 \mathrm{a}$ an optimal pattern is presented where the gap between P1-P2 and P2-P3 and thus the loss due to inactive area is minimized. In this case the width covered by P2, P3, and the space between the scribed lines is $200 \mu \mathrm{m}$. While the former pattern depicts the optimum achievable with the mechanical scribing setup, a more favorable pattern is presented in Fig. 4b. Here, the gaps between P1-P2 and P2-P3 permit a higher lateral tolerance of the scribed lines allowing for higher patterning speeds. Therefore, this design is applied in the standard module fabrication process. As it can be seen in both images, the width of the mechanical scribed lines varies between $70 \mu \mathrm{m}$ and $85 \mu \mathrm{m}$. While narrower lines can be realized experimentally their negative impact on yield and device performance prevents them from a successful application. Overall, the total width loss due to the three patterning steps is around $300 \mu \mathrm{m}$ resulting in a geometrical fill factor of $95 \%$. Here, the term geometrical fill factor defines the ratio of active area to the aperture area of $16 \mathrm{~cm}^{2}$.

\subsection{Mechanically stacked modules}

Figure 5 shows the sample architecture of a tandem device formed by two mechanically stacked devices in a semitransparent configuration. In the presented design, the light incidence occurs from the bottom side which is the substrate side of a semitransparent PV10 device. The second submodule is stacked with its active layer side containing as active material XPbing onto the bottom submodule. For an improved internal light absorption the backside of the top submodule is coated with a thick intransparent and highly reflective Ag film. The two submodules are mechanically connected by an edge sealing with encapsulation glue while keeping them electrically separated.

The presented configuration is the result of a computational optimization considering the optical and electrical properties of the thin film devices. Here, the PV10 submodule is efficiently harvesting the shorter wavelength of the module absorption range while the XPbing device is contributing mainly with the near infrared absorption to the total power output. Reflected light from the XPbing module can increase the power generation in the PV10 subdevice additionally. 


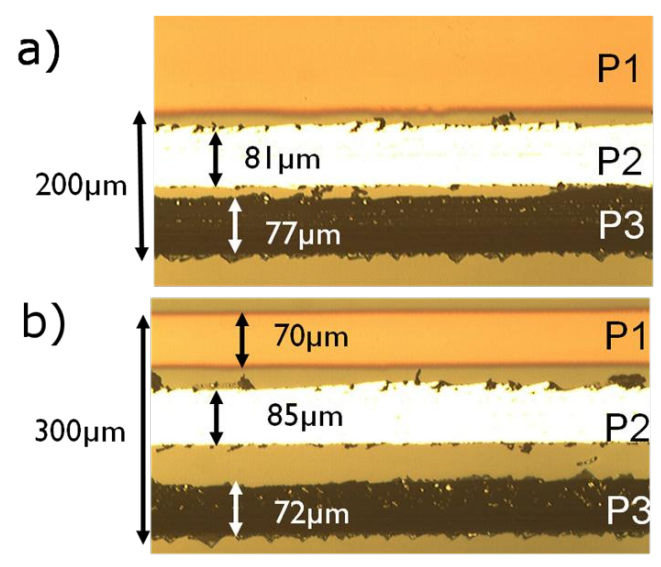

Figure 4 .

Optical microscope images of patterned organic solar modules recorded in reflection. The top image shows the P2 and P3 scribes running with minimal area loss along the edge of the ITO contact (a).The loss of the active area on the $5 \mathrm{~mm}$ ITO stripe width is minimized to $200 \mu \mathrm{m}$. In the bottom image the P1 lithography pattern between two ITO stripes is shown together with P2 and P3 scribes that leave more space to increase yield at higher scribing speeds (b). The active area loss by the three patterning steps adds up to $300 \mu \mathrm{m}$ of the $5 \mathrm{~mm}$ ITO line width resulting in a geometrical fill factor of $95 \%$.

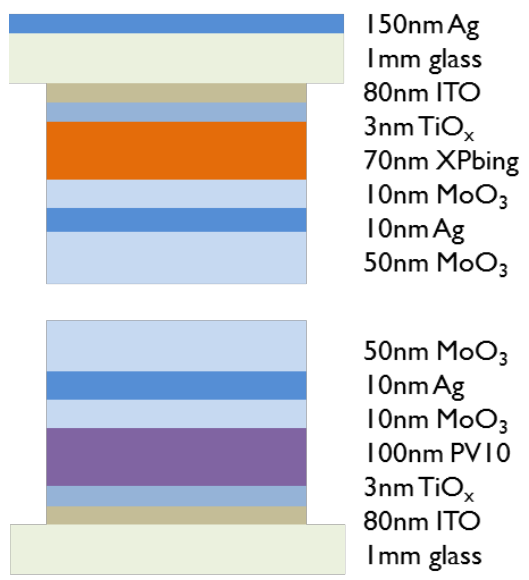

Figure 5 .

Structure and layer thicknesses of a mechanically stacked tandem device. In this configuration the light is incident from the bottom.

\section{RESULTS}

\subsection{Monolithic modules}

As presented in the previous section, the finally stacked tandem modules consist of two semitransparent submodules. In Fig. 6 the J-V curves of the best semitransparent XPbing and PV10 modules are presented. In this direct comparison the higher performance of the PV10 module is evident with higher values in short circuit current density, open circuit voltage, and fill factor. Table 2 lists the electrical parameters of the same semitransparent XPbing and PV10 modules versus the best opaque PV10 module* ${ }^{\dagger}$. As expected, the semitransparent PV10

\footnotetext{
${ }^{*}$ A similar device has been externally certified by Newport with $5.5 \%$ aperture efficiency.

${ }^{\dagger}$ For single cells it is common practice to present the short circuit current density instead of the current. In serially connected modules the extracted current is equal to the current generated by the least efficient subcell. We present the current density as it is derived by Jsc = current of module/aperture area. Multiplying this Jsc with the number of subcells (here 8) gives the current density within a subcell. Accordingly is the Voc of a module presented by giving the value of
} 
Table 2. Electrical properties of monolithic PV10 and XPbing modules with aperture areas of $16 \mathrm{~cm}^{2}$. An opaque PV10 device with intransparent $150 \mathrm{~nm}$ Ag layers is compared to semitransparent modules with $10 \mathrm{~nm}$ Ag containing either PV10 or XPbing as active material. All devices have been illuminated through the glass substrate. For an easier comparison with the single cells of Tab. 1 the values of the Jsc and the Voc in the brackets represent the equivalent values of a single cell in the modules by a correction according to the number of serially connected subcells. The presented efficiencies are calculated considering the aperture area of $\mathrm{A}=16 \mathrm{~cm}^{2}$.

\begin{tabular}{|c|c|c|c|c|c|}
\hline active material & configuration & Jsc $(\mathbf{8} \times \mathbf{J s c})\left[\mathrm{mA} / \mathrm{cm}^{2}\right]$ & Voc $(\mathbf{V o c} / \mathbf{8})[\mathrm{mV}]$ & $\mathbf{F F}[\%]$ & Eff $[\%]$ \\
\hline PV10 & opaque & $1.42(11.4)$ & $5880(740)$ & 63 & 5.2 \\
\hline PV10 & semitransparent & $1.18(9.4)$ & $6270(780)$ & 67 & 5.0 \\
\hline XPbing & semitransparent & $1.01(8.1)$ & $5520(690)$ & 49 & 2.8 \\
\hline
\end{tabular}

module is slightly less efficient than its equivalent with the highly reflective Ag contact. This lower efficiency is caused by a reduced short circuit current density. The reason that the difference is much smaller as for the small area devices in Tab. 1 is the increase in open circuit voltage and a higher fill factor of the semitransparent module. This result confirms the good electrical performance of the $\mathrm{MoO}_{3} / \mathrm{Ag} / \mathrm{MoO}_{3}$ top contact. Compared to the small area single cells the semitransparent modules have about $80 \%$ of the efficiency. While for PV10 the module performance loss is solely caused by a reduction of the Jsc, the reduced relative power of the XPbing module is caused by a Jsc drop and a fill factor reduction, equally.

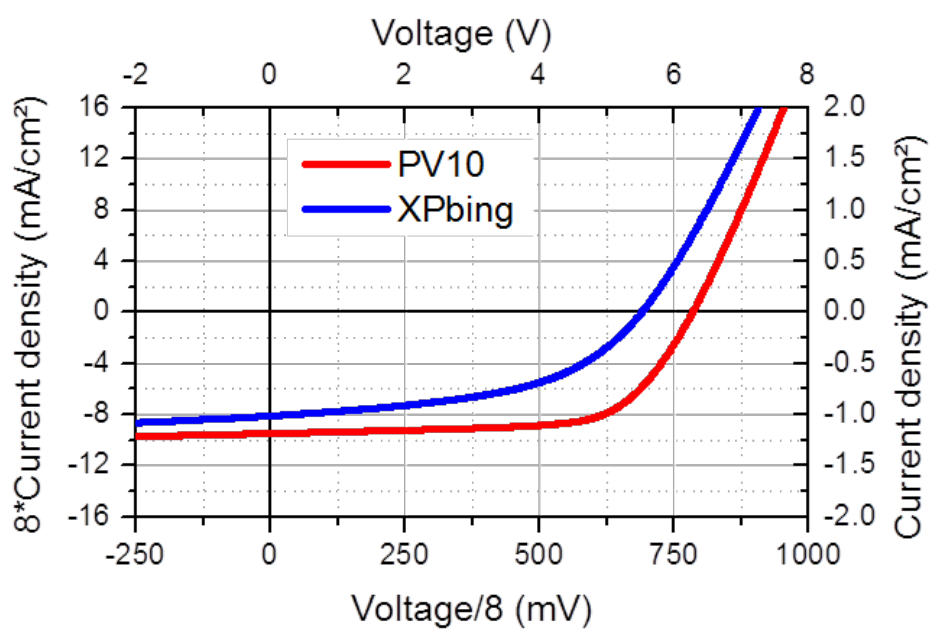

Figure 6 .

$\mathrm{J}-\mathrm{V}$ characteristic of semitransparent modules with the active materials containing the polymers PV10 and XPbing. The modules consist of 8 subcells connected in series. For this reason the axes show the measured values of voltage and current density as well as the normalized values for easy comparison with single cells.

The optical properties of the semitransparent devices are depicted in a comparison of the optical transmission (Fig. 7(a)) and the resulting color coordinates of incident white light after transmission (Fig. 7(b)). While the high absorption of the thicker PV10 film results in a transmittance from 10-20\% in the visual spectral range, the XPbing device has a generally higher transmittance which peaks with around $50 \%$ at $600 \mathrm{~nm}$ in the range of visible light. The larger transmission intensity variation of the XPbing module leads to a slightly larger color shift towards the orange part of the spectrum as it is also visualized in the CIE diagram. An important

the module and the voltage devided by the number of subcells representing the average voltage generated by a subcell. 
optical aspect of the PV10 module corresponding to the application in stacked tandem cells is the transmission window for light with wavelength longer than $700 \mathrm{~nm}$. The transmission of $40-50 \%$ is much lower than expected from optical simulations but still large enough to support the operation of an XPbing device in a mechanically stacked tandem configuration. The low long wavelength transmittance of the PV10 device is mainly caused by a misalignment of the layers in the $\mathrm{MoO}_{3} / \mathrm{Ag} / \mathrm{MoO}_{3}$ top contact, as it is supported by a computational analysis of the optical measurements.
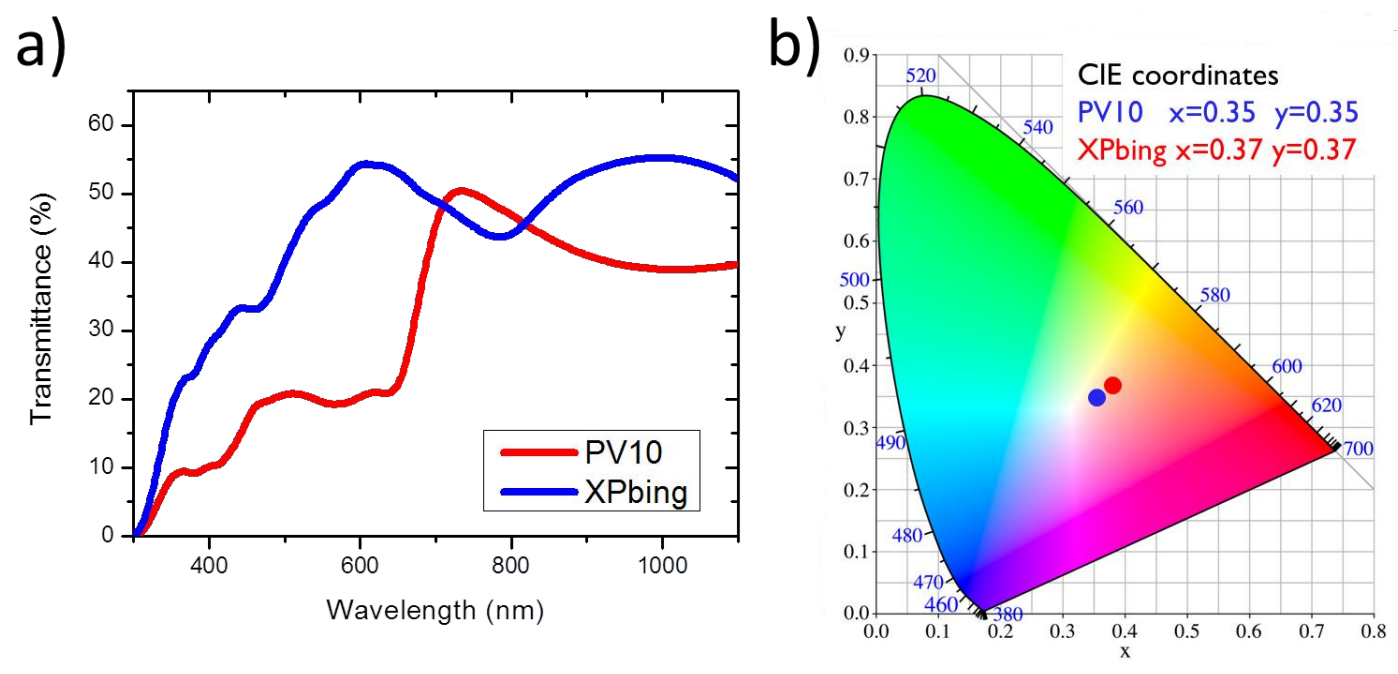

Figure 7 .

Measured transmittance of semitransparent modules containing PV10 (red) and XPbing (blue) as donor polymer in the active layer (a). The CIE color coordinates of the two transmission spectra after white light illumination are closely located to the white point $(\mathrm{x}, \mathrm{y}=0.33)$.

Semitransparent OPV modules are good candidates for the integration in glass facades for reduced light transmission and energy harvesting. The photographic image in Fig. 8 demonstrates the optical appearance of a PV10 module which is similar to conventional glass coatings based on reflection and absorption. The low transmission of 10-20\% allows for the application under conditions where a higher absorption is preferred.

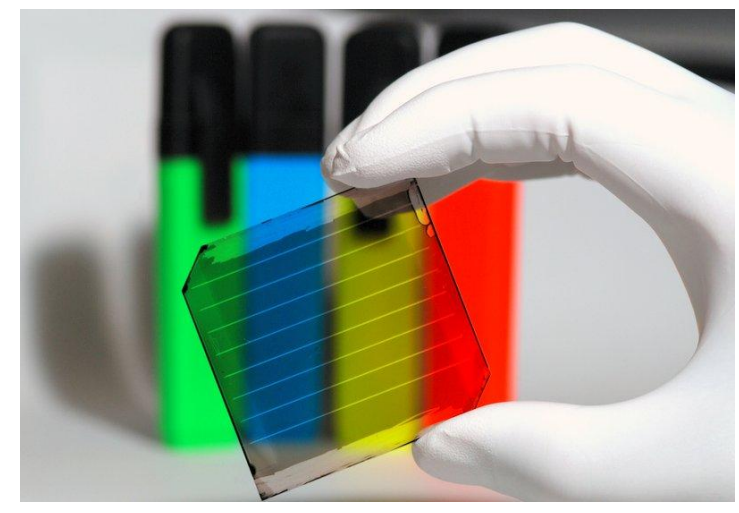

Figure 8.

Photographic image of a semitransparent PV10 module illustrating the low color shift of transmitted light. The presented module had an initial efficiency of around $5 \%$ under inert atmospheric conditions. 


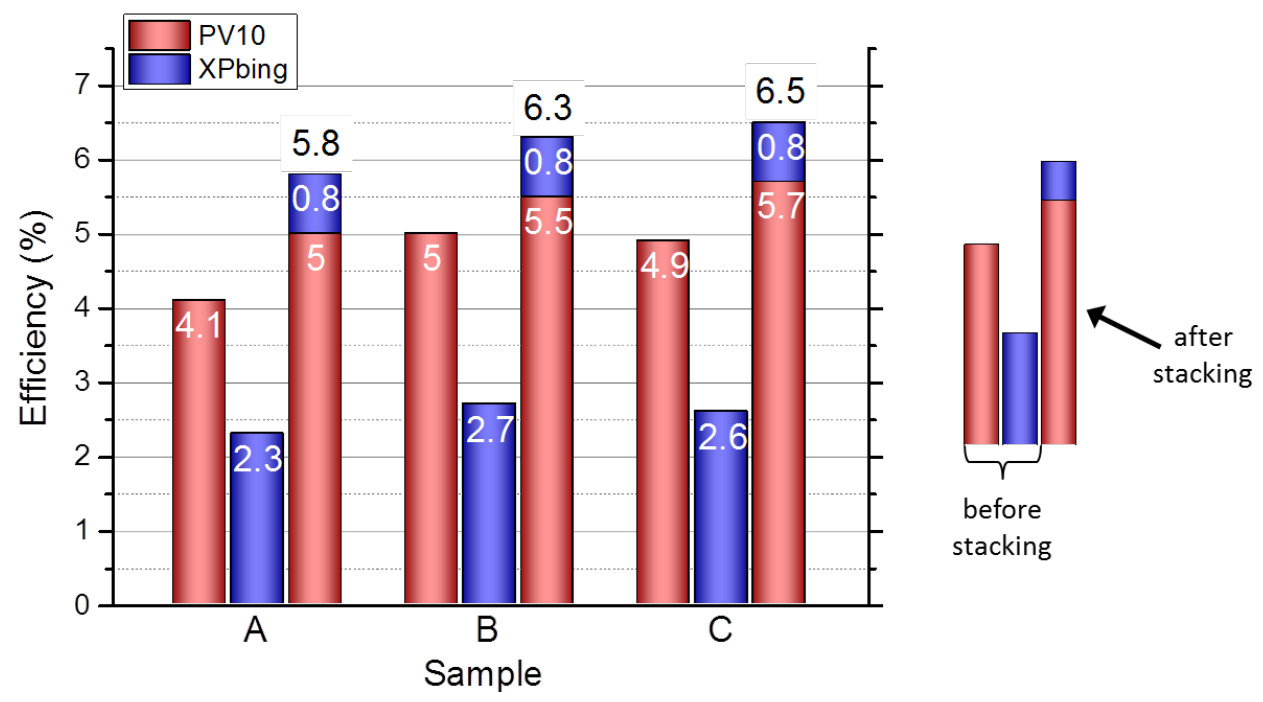

Figure 9.

Comparison of module efficiencies before and after mechanical stacking. For three samples the efficiencies of the semitransparent submodules before and after stacking are shown. In all samples the PV10 module efficiency increases after integration in the module assembly. Due to the absorption of the first cell in the stack the XPbing power output drops to $0.8 \%$ in all samples. The two best performing tandem stacks have efficiencies of $6.3 \%$ and $6.5 \%$, respectively.

\subsection{Mechanically stacked module assembly}

For the final assembly of mechanically stacked module combinations the best semitransparent PV10 and XPbing modules have been selected. Before combining two modules, a $150 \mathrm{~nm} \mathrm{Ag} \mathrm{film} \mathrm{has} \mathrm{been} \mathrm{deposited} \mathrm{on} \mathrm{the} \mathrm{backside}$ of the XPbing modules in order to enhance backside reflection and thus the overall module performance. Figure 9 compares for three stacked samples the efficiencies of the modules before the assembly with the power output after mechanical combination. The presented efficiencies for the XPbing modules represent the data before the back side coating. For the PV10 modules an efficiency increase between $10 \%$ and $20 \%$ is observed while the XPbing efficiencies drop from around $2.5 \%$ to $0.8 \%$. For the later devices this is expected as the largest spectral part of the XPbing absorption is covered by the PV10 modules which are located on the direction of light incidence. Assuming a complete absorption of incident light within the PV10 absorption band and a full transmission of the remaining spectral range, the XPbing efficiency of $0.8 \%$ meets the expectations. The increase of the power efficiency of the PV10 submodules is partly explained by an optical improvement due to back reflections from the XPbing submodules. A second reason for the improvement is caused by the device aging of the inverted structure as it has been observed for most of the devices within this work and is related to modifications in the oxide films and the corresponding interfaces. Since the final module stacks are four-terminal devices, the power of each submodule is extracted and measured in separate electrical circuits. Both submodules are illuminated through the same aperture area which allows for an addition of the two effiencies. In the three presented samples the overall power efficiency ranges from $5.8 \%$ to $6.5 \%$, thus excelling the maximum efficiencies of the most efficient opaque PV10 modules by $25 \%$.

Figure 10 depicts the $\mathrm{J}-\mathrm{V}$ characteristic and the power output of the submodules in the best stacked device. From both graphs it is evident that current densities and voltages at the maximum power points of the two submodules differ, preventing from an efficient parallel or serial connection of the two devices to transform the module stack from a four-terminal device into a two-terminal photovoltaic cell. By applying different subcell patterns for the two submodules this problem can be overcome and two terminal devices based on this construction principle can be fabricated. Unfortunately, every design would be optimized for a narrow range of illumination conditions considering variations in the spectrum and angle of the incident light. Therefore, the most promising design will be based on a four-terminal architecture were the power is harvested separately. The currents and voltages are going to be transformed externally before further transmission and application. 
a)

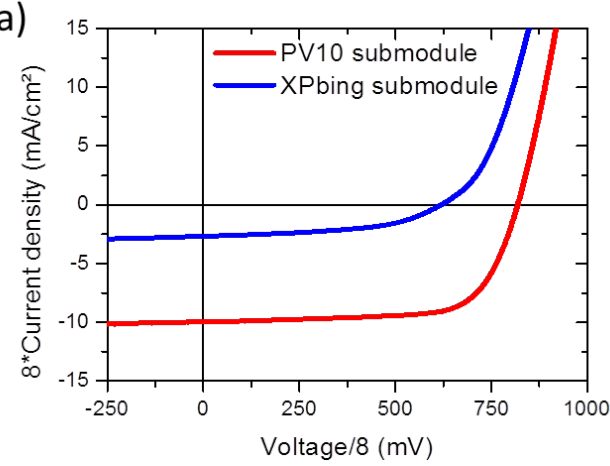

b)

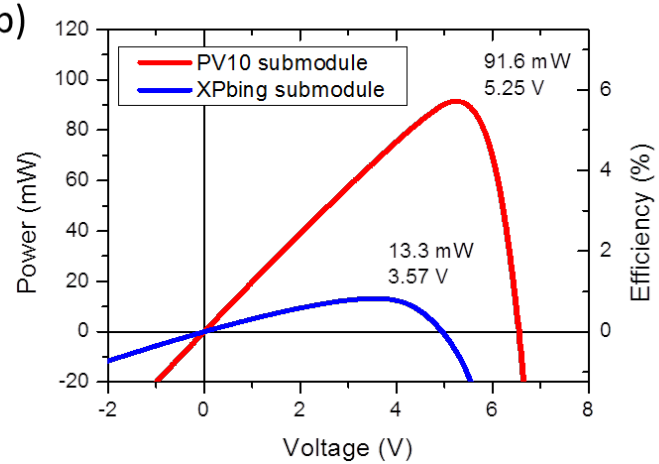

Figure 10.

$\mathrm{J}-\mathrm{V}$ characteristic (a) and power output (b) of the submodules of a mechanically stacked tandem device. Both submodules use the same aperture. The submodules were measured individually and subsequently within a second. The added power of about $105 \mathrm{~mW}$ results in an aperture power efficiency of over $6.5 \%$.

\subsection{Optical simulation}

In order to evaluate the potential of the presented concept of mechanically stacked modules, we benchmark series connected tandem cells with the four-terminal cells. The following computational procedure is taken:

- optimization of the layer structure

- calculation of the external quantum efficiencies for the subcell for incident angle from $0^{\circ}$ to $90^{\circ}$

- apply corresponding solar air mass spectra for different sun elevations, both for direct and diffuse light (obtained from NREL)

- apply recorded solar irradiance data of a central European location $\ddagger$

- using blocks of one hour to calculate the position of the sun and the incident angle on the devices for every possible moment in the year for a solar cell directed south under a certain vertical angle

- compute the photocurrent and the related produced power of the OPV devices for every possible moment in the year

- calculate a yearly produced power in dependence on the mounting angle of the cell.

The first calculations use a series connected tandem architecture, with PV10 as the front, and XPbing as the back cell. The complete structure is optically optimized using simulations based on a transfer matrix algorithm. In such a structure, the produced photocurrent is limited by the lowest photocurrent of the subcells, the open circuit voltage is the sum of the individual voltages, and the fill factor is the lower value of the two subcells.

Simulated EQE spectra are shown in Fig. 11 (top), for both subcells. As can be observed in both cases, the EQE signal shows only slight dependencies on the angle of incidence. For both subcells the most prominent changes are observed for the wavelength range from $450 \mathrm{~nm}$ to $650 \mathrm{~nm}$, which is caused by a change in the optical interference pattern and by an increased optical path inside the organic materials, which compensates the reduced light incoupling due to reflection at the air-glass interface.. For normal incidence, the subcells have peak EQE values close to $60 \%$ for PV10 and $45 \%$ for XPbing, with complementary features.

\footnotetext{
${ }^{\ddagger}$ Due to its availability, the data of Eindhoven, Netherlands $\left(51^{\circ} 26^{\prime} \mathrm{N} 05^{\circ} 28^{\prime} \mathrm{E}\right)$ for the period of 3 years have been used.
} 


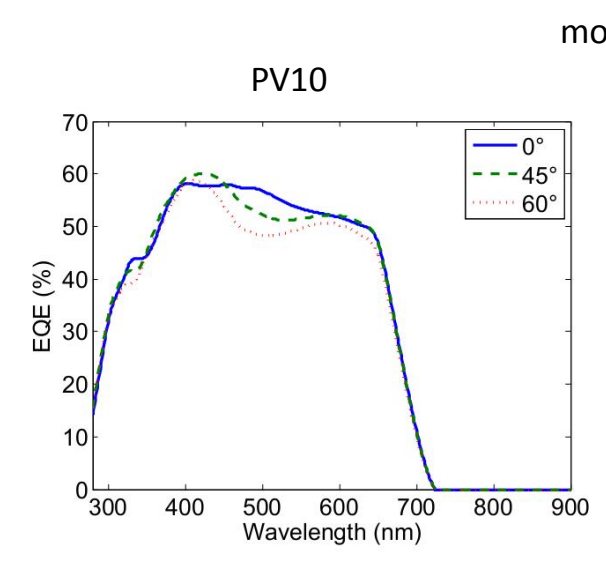

monolithic tandem

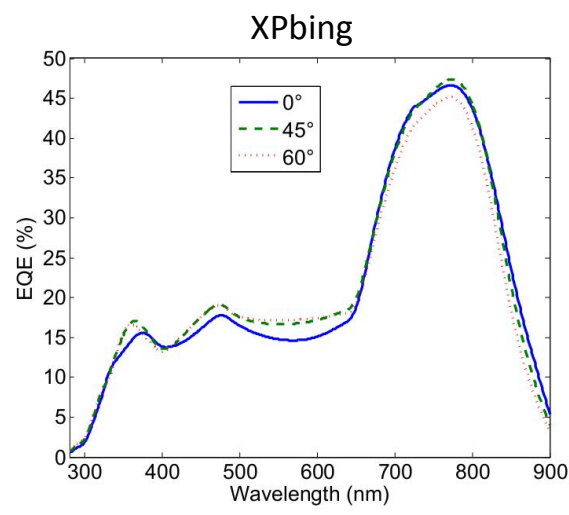

mechanically stacked tandem
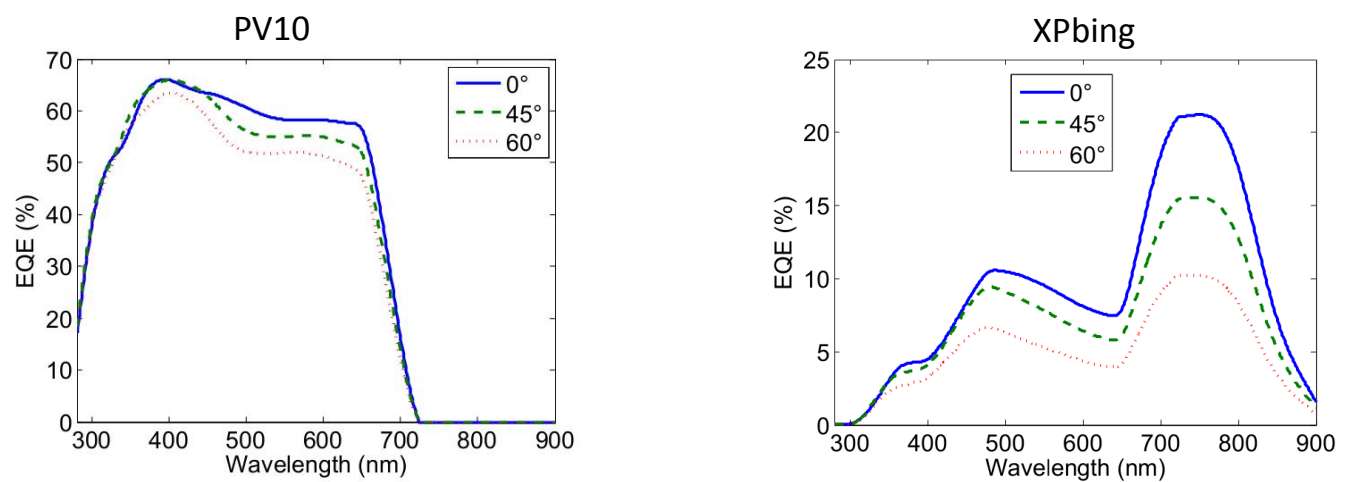

Figure 11.

EQE values of a series connected monolithic tandem cell (top) and the mechanically stacked tandem (bottom), as simulated using transfer matrix. For three different incident angles the EQE is shown for the corresponding PV10 subcell (left) and the XPbing subcell (right).

The same simulations are done for a mechanically stacked device. For these simulations, both subcells are treated as incoherent with each other, as well as with the silver mirror on the backside of the glass substrate. The results can be seen in Fig. 11 (bottom). The optimized structure uses a thick PV10 based subcell that will produce most of the photocurrent, and a thinner XPbing subcell atop. The EQE spectra look vastly different: PV10 exhibits peaks up to $65 \%$, while XPbing shows a maximum EQE of $21 \%$. Both subcells exhibit a stronger angle dependence as the previously simulated monolithic devices. Especially the XPbing device shows a strong EQE decrease over the whole spectrum with an increasing angle of incidence. However, within the four-terminal device concept, this does not lead to a major problem, as there are no restrictions regarding the connections (series-parallel). A more critical loss indicated by the simulations is the light absorption within the transparent thin silver contacts with $15-20 \%$ of the incident intensity. This fact needs closer attention in future optimizations of the concept.

Table 3 shows the current mismatch in a monolithic tandem cell by applying the same modeling algorithm as used before. Here, the angle of incidence and the irradiation light spectra have been varied. Furthermore, the values for diffuse illumination are presented. The mismatch between the currents produced by the subcells is showing only a small angular dependence with variations between $10 \%$ and $20 \%$ for the three different spectra. However, the mismatch is remarkably increasing for diffuse light by reaching up to $66 \%$ for the AM1.5 diffuse illumination spectrum. This mismatch is clearly limiting the electrical power output of such a device in locations or orientations where diffuse illumination is the dominating type of incident radiation, as the excess carriers generated will recombine within the layer structure. 
Table 3. Simulated short-circuit current mismatch for an optically optimized series connected monolithic tandem cell. The simulation is done for three different solar spectra, and three different incident light angles as well as for diffuse light.

\begin{tabular}{|c|c|c|}
\hline spectrum & incident angle & mismatch [\%] \\
\hline \multirow{3}{*}{ AM1 } & $0^{\circ}$ & 22 \\
& $45^{\circ}$ & 19 \\
& $60^{\circ}$ & 18 \\
& diffuse & 44 \\
\hline \multirow{3}{*}{ AM1.5 } & $0^{\circ}$ & 18 \\
& $45^{\circ}$ & 15 \\
& $60^{\circ}$ & 13 \\
AM2 & diffuse & 66 \\
\hline & $0^{\circ}$ & 13 \\
& $45^{\circ}$ & 9 \\
& $60^{\circ}$ & 10 \\
\hline
\end{tabular}

The same simulation algorithm is applied in the calculations of the annually produced photocurrent of the mechanically stacked device for every possible moment of the year, by using recorded sun irradiation data for Eindhoven. Figure 12 compares the annual output power of the optically optimized monolithic tandem solar cell with the mechanically stacked four-terminal structure. Both device structures show in the simulation an equal efficiency of $6.6 \%$ for lab conditions with normal incidence of direct AM1.5 illumination. The simulation of the annual energy production shows the results in dependence on the tilting angle of solar modules facing South. Both devices show the maximum energy output at tilting angles of $34^{\circ}$ with the higher generated energy for the four-terminal device. Comparing the values at the optimal tilting angle the stacked cell architecture produces about $32 \%$ more energy over the course of one year than the monolithic tandem. With an annual solar incidence of $1050 \mathrm{kWh} / \mathrm{m}^{2} \mathrm{a}$ at the chosen location the mechanically stacked module produces an energy of around $108 \mathrm{kWh} / \mathrm{m}^{2}$ a resulting in an overall annual efficiency of over $10 \%$. In comparison, the monolithic module has an annual efficiency of $7.8 \%$ in this simulation.

The reason for the advantage of the four-terminal device is the previously mentioned current mismatch of the subcells in the monolithic tandem device. While in the stacked device about $20 \%$ of the incoming light is lost by absorption in the transparent contacts, even under direct illumination $10 \%$ to $20 \%$ of the direct incident radiation is lost in a conventional tandem by unbalanced currents. Under diffuse light illumination, which is the dominating incident power at overcast weather conditions or larger azimuth angles of the sun, the imbalance of the generated number of charges in the subcells excels the optical losses in the four-terminal structure. Under the applied simulation conditions, the four-terminal concept clearly outperforms the conventional monolithic tandem architecture.

\section{CONCLUSION}

The results presented within this work show the potential of the four-terminal architecture to increase the annual electrical energy yield over series connected devices as used in conventional tandem concepts. Optical simulations of the new device structure, experimentally exhibiting 6.5\% efficiency under vertical illuminatiuon with an AM1.5 spectrum, excel the energy output of a standard tandem device with the same organic absorber systems by more than $32 \%$ over a year considering central European solar irradiance conditions. Especially the independence on balancing the currents or voltages of tandem subcells eliminates the factor of spectral dependencies. Furthermore the selected absorber materials do not need to be completely complementary in their absorption spectra as there is no requirement of producing an equal number of charges from different parts of the optical spectrum. Here, it has been shown for one set of materials, that two subcells with slightly different absorption spectra can be combined to an efficient device. The reduced dependence on the optical parameters of the materials allows for a selection on other specifications as, for example, film processibility or thermal and light stability. A future 


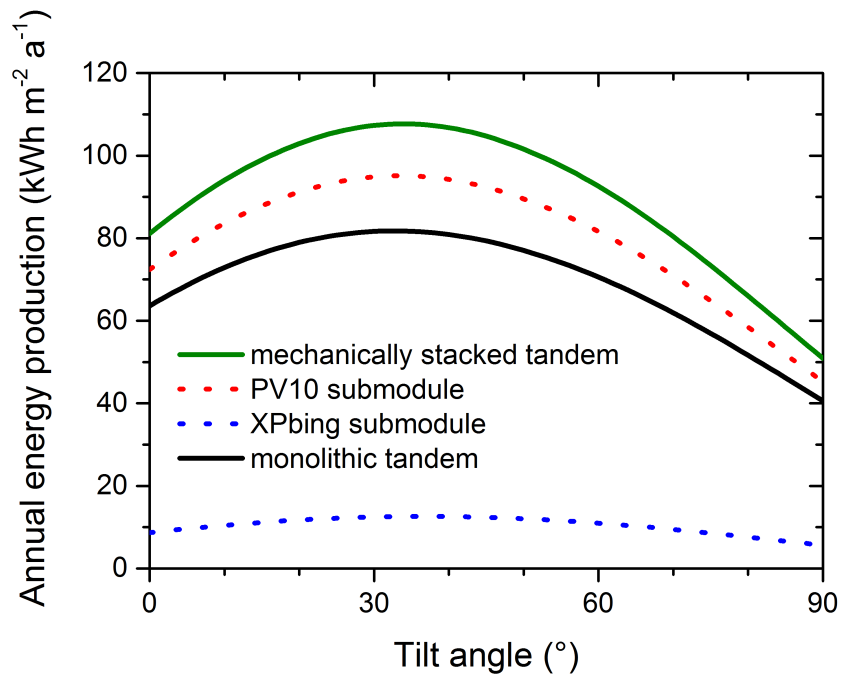

Figure 12.

Comparison of the annual energy yield of an optimized monolithic tandem cell and a mechanically stacked device using real sun irradiance data for Eindhoven, Netherlands $\left(51^{\circ} 26^{\prime} \mathrm{N} 05^{\circ} 28^{\prime} \mathrm{E}\right)$ considering direct and diffuse sunlight. For the mechanically stacked device the power generation of the PV10 and XPbing submodules is plotted additionaly.

optimization of the material components for the submodule absorbing in the longer wavelength range offers a large potential for an increase in the power output. Recently, efficient organic compounds and devices of small molecules and polymers have been presented with high EQEs up to wavelength of $1000 \mathrm{~nm} .^{4,8,9}$ Considering the small contribution of the currently used small bandgap material to the overall power output a remarkable increase of efficiency can be expected. Furthermore it has to be stated that a large fraction of incident light is lost by absorption in the top contacts of the submodules. A loss reduction on this device section will additionally enhance the stack performance.

Within the course of this work semitransparent single junction devices have been developed. With efficiencies of $5.0 \%$ on a module level, these devices show a similar performance as their opaque counterparts. In contrast to the opaque mechanically stacked modules with their target of most efficient light harvesting, these semitransparent devices are good candidates for and application in roof tiles and glass facades. With their low color shift they offer new opportunities in architectural design.

\section{REFERENCES}

[1] Heliatek GmbH. Press release (January 16, 2013). www.heliatek.com.

[2] Green, M. A., Emery, K., Hishikawa, Y., Warta, W., and Dunlop, E. D., "Solar cell efficiency tables (version 42)," Progress in Photovoltaics: Research and Applications 21(5), 827-837 (2013).

[3] Cheyns, D., Rand, B. P., and Heremans, P., "Organic tandem solar cells with complementary absorbing layers and a high open-circuit voltage," Applied Physics Letters 97(3), 33301 (2010).

[4] You, J., Dou, L., Yoshimura, K., Kato, T., Ohya, K., Moriarty, T., Emery, K., Chen, C.-C., Gao, J., Li, G., and Yang, Y., "A polymer tandem solar cell with $10.6 \%$ power conversion efficiency," Nature Communications 4, 1446 (2013).

[5] Gilot, J., Wienk, M. M., and Janssen, R. A. J., "Optimizing polymer tandem solar cells," Advanced Materials 22(8), E67-E71 (2010).

[6] Hadipour, A., Müller, R., and Heremans, P., "Room temperature solution-processed electron transport layer for organic solar cells," Organic Electronics 14(10), 2379 - 2386 (2013). 
[7] Sergeant, N. P., Hadipour, A., Niesen, B., Cheyns, D., Heremans, P., Peumans, P., and Rand, B. P., "Design of transparent anodes for resonant cavity enhanced light harvesting in organic solar cells," Advanced Materials 24(6), 728-732 (2012).

[8] Vasseur, K., Rand, B. P., Cheyns, D., Froyen, L., and Heremans, P., "Structural evolution of evaporated lead phthalocyanine thin films for near-infrared sensitive solar cells," Chemistry of Materials 23(3), 886-895 (2011).

[9] Li, W., Furlan, A., Hendriks, K. H., Wienk, M. M., and Janssen, R. A. J., "Efficient tandem and triplejunction polymer solar cells," Journal of the American Chemical Society 135(15), 5529-5532 (2013). 